\title{
PHASE PORTRAITS OF PLANAR SEMI-HOMOGENEOUS VECTOR FIELDS (III)
}

\author{
LAURENT CAIRÓ AND JAUME LLIBRE
}

\begin{abstract}
We determine all the phase portraits of the semi-homogeneous polynomial vector fields of the form $\left(a x+b y, A x^{4}+B x^{3} y+C x^{2} y^{2}+D x y^{3}+\right.$ $\left.E y^{4}\right)$.
\end{abstract}

\section{INTRODUCTION}

Let $P$ and $Q$ be real homogeneous polynomials in the variables $x$ and $y$ of degrees $m$ and $n$ respectively. Then we say that $X=(P, Q): \mathbb{R}^{2} \longrightarrow \mathbb{R}^{2}$ is a semihomogeneous polynomial vector field, in particular if $m=n, X$ is a homogeneous polynomial vector field.

The case $m=n$ has been studied by several authors. The homogeneous quadratic polynomial vector fields $(m=n=2)$ by Lyagina [16], Markus [17], Korol [13], Sibirskii and Vulpe [19], Newton [18], Date [11] and Vdovina [20]; the homogeneous cubic polynomial vector fields $(n=m=3)$ by Cima and Llibre [8]; the general case $m=n$ by Argemí [4], Cima and Llibre [8], Collins [10], Llibre, Pérez del Río and Rodriguez $[14,15]$. In these papers is described an algorithm for studying the phase portraits of homogeneous polynomial vector fields for all degree $m=n$, the classification of all phase portraits of homogeneous polynomial vector fields of degree 2 and 3, the algebraic classifications of homogeneous polynomial vector fields and the characterization of structurally stable homogeneous and semi-homogeneous polynomial vector fields. In a previous papers $[6,7]$, we studied the phase portraits of the semi-homogeneous vector fields with $m=1, n=2$ and $m=1, n=3$ respectively.

The main goal of this paper is to study the semi-homogeneous vector fields of degrees $m=1$ and $n=4$ (i.e. vector fields $\left(a x+b y, A x^{4}+B x^{3} y+C x^{2} y^{2}+D x y^{3}+\right.$ $\left.E y^{4}\right)$ with $|a|+|b| \neq 0$ and $|A|+|B|+|C|+|D|+|E| \neq 0$ ) having finitely many singular points. The classification of the phase portraits of these vector fields is also interesting as a subclass of the quartic polynomial vector fields. We note that these polynomial differential systems has no limit cycles, [9] or [15].

Our main result is the following one.

Theorem 1. The phase portrait of any planar semi-homogeneous polynomial vector field of degrees 1 and 4 having finitely many singular points is topologically

1991 Mathematics Subject Classification. Primary 34C05, 34A34, 34C14.

Key words and phrases. semi-homogeneous vector fields, phase portraits.

The second author is partially supported by a DGICYT grant number MTM2005-06098-C02-01 and by a CICYT grant number 2005SGR 00550. 

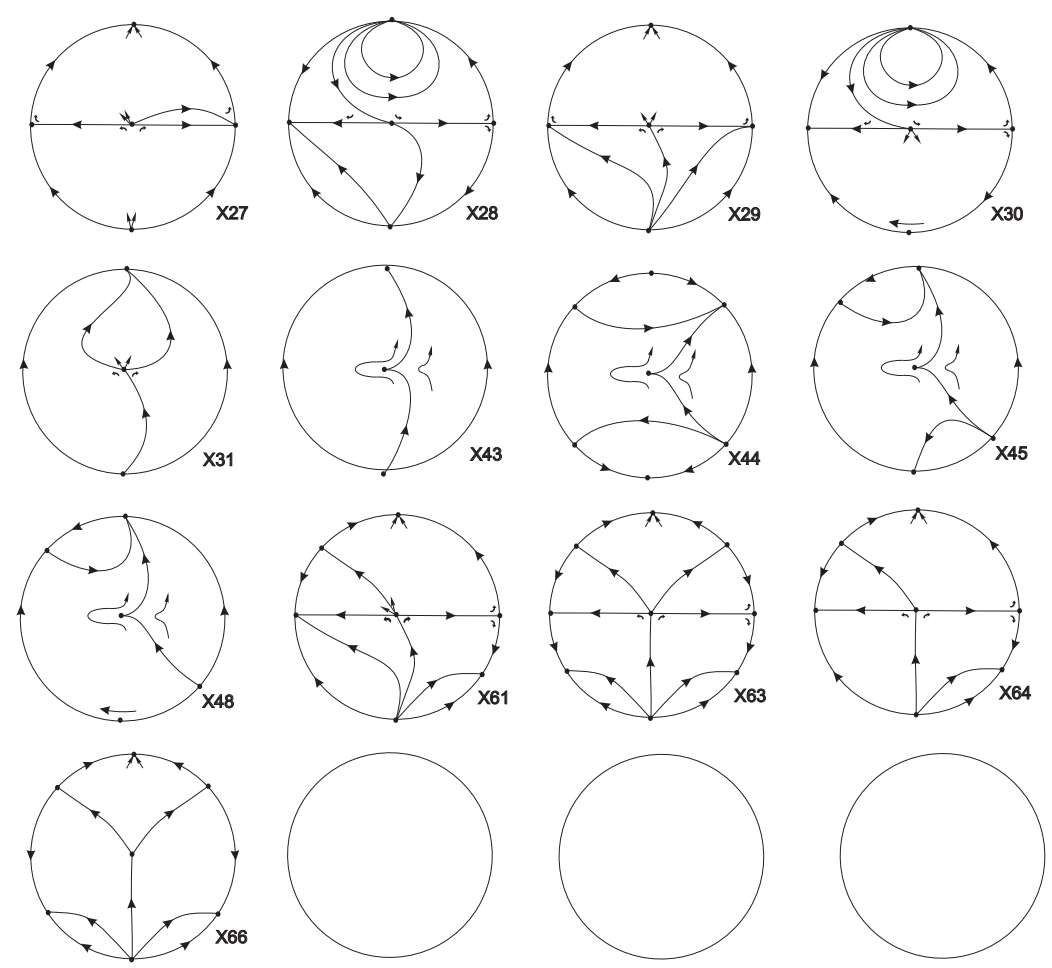

Figure 1. The $X$ non topologically equivalent phase portraits of a semi-homogeneous polynomial vector field of the form $(a x+$ by, $\left.A x^{4}+B x^{3} y+C x^{2} y^{2}+D x y^{3}+E y^{4}\right)$.

equivalent to one of the ?? configurations of Figure 1. Moreover each of the configurations of Figure 1 is realizable for a planar semi-homogeneous polynomial vector field of degree 1 and 4 .

The topological equivalence in Theorem 1 is defined for the compactified vector fields, see Subsection 4.

The paper is organized as follows. In Section 2 we reduce the initial six parameters of a planar semi-homogeneous polynomial vector field $X=\left(a x+b y, A x^{4}+\right.$ $\left.B x^{3} y+C x^{2} y^{2}+D x y^{3}+E y^{4}\right)$ to 67 normal forms of at most four parameters. The basic definitions and results about the different kinds of finite and infinite singular points are given in Section 3. The local phase portraits of the finite and infinite singular points of our semi-homogeneous vector fields are described in Sections 4 and 5 respectively. Finally the local and global phase portraits of our semi-homogeneous vector fields are described in Sections 6 and 7, respectively.

\section{NORMAL FORMS}

Our interest here is localized in the semi-homogeneous vector fields $X=(P, Q)$ of the form $P=a x+b y$ linear and $Q=A x^{4}+B x^{3} y+C x^{2} y^{2}+D x y^{3}+E y^{4}$ quartic having finitely many singular points. Such a semi-homogeneous vector 
field has seven independent coefficients. In this section we prove that we can reduce the study of these systems to 67 normal forms, taking into account the essential parameters.

We say that two vector fields $X$ and $Y$ on $\mathbb{R}^{2}$ are topologically equivalent if there exists a homeomorphism of $\mathbb{R}^{2}$ carrying orbits of the flow induced by $X$ into orbits of the flow induced by $Y$, preserving or reversing simultaneously the sense of all orbits.

Proposition 2. Let $X$ be a semi-homogeneous vector field of the form (ax + by, $\left.A x^{4}+B x^{3} y+C x^{2} y^{2}+D x y^{3}+E y^{4}\right)$ having finitely many singular points, then its phase portrait is topologically equivalent to one of the phase portraits of the vector fields of the following list:

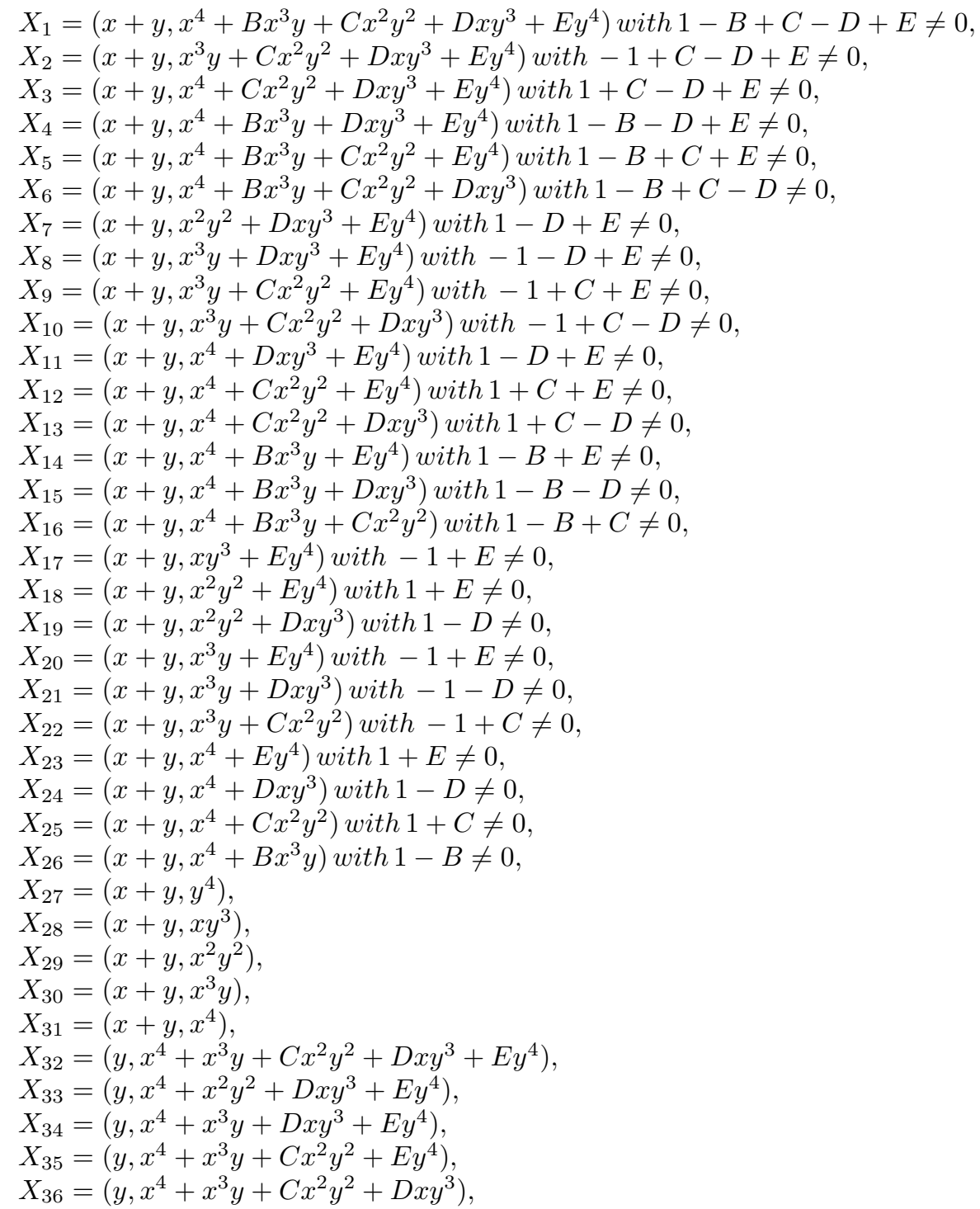




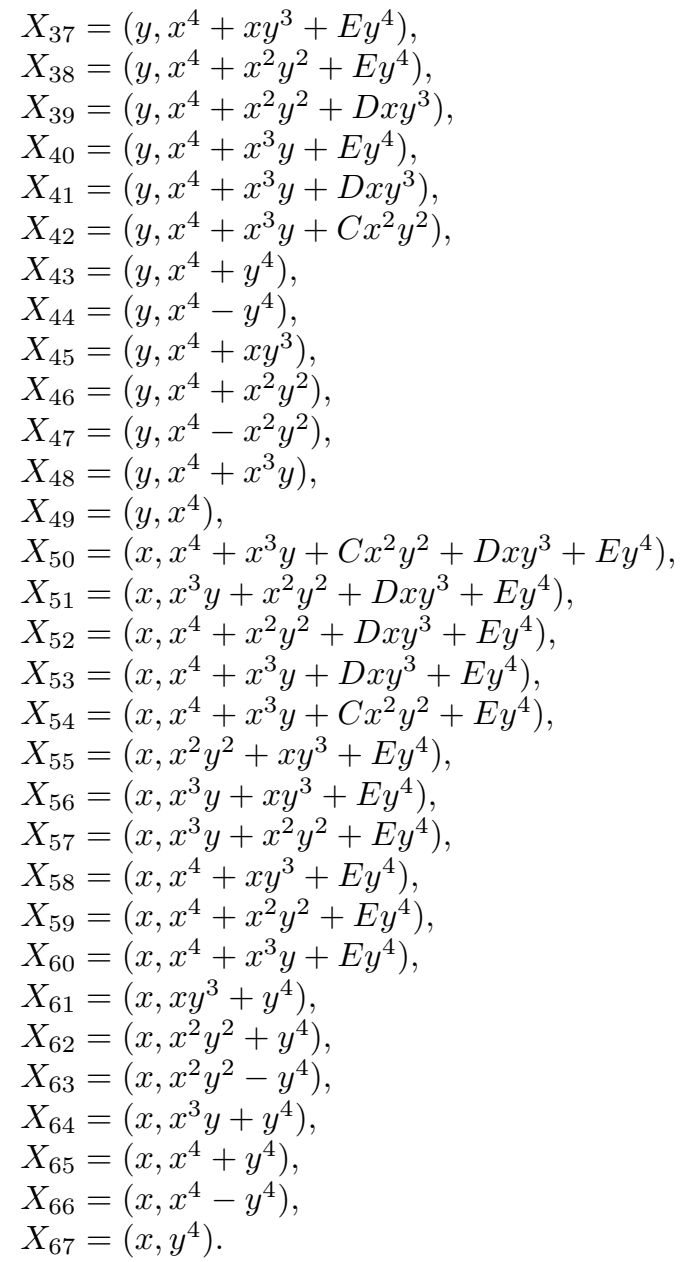

If in the expression of $X_{i}$ appears some of the parameters $B, C, D$ or $E$, these are always non-zero.

Proof. Let $\bar{x}=\alpha x, \bar{y}=\beta y, \bar{t}=\gamma t$. Then $X$ writes in the transformed variables $\left(a \bar{x} / \gamma+\alpha b \bar{y} /(\beta \gamma), \beta A \bar{x}^{4} /\left(\alpha^{4} \gamma\right)+B \bar{x}^{3} \bar{y} /\left(\alpha^{3} \gamma\right)+C \bar{x}^{2} \bar{y}^{2} /\left(\alpha^{2} \beta \gamma\right)+D \bar{x} \bar{y}^{3} /\left(\alpha \beta^{2} \gamma\right)+\right.$ $\left.E \bar{y}^{4} /\left(\beta^{3} \gamma\right)\right)$.

The 67 different normal forms appear when we consider the combination of the three possibilities of $a$ and $b(a b \neq 0 ; a=0$ and $b \neq 0 ; a \neq 0$ and $b=0)$ with the thirty one possibilities of $A, B, C, D$ and $E(A B C D E \neq 0, A=0$ and $B C D E \neq 0$, $B=0$ and $A C D E \neq 0, C=0$ and $A B D E \neq 0, D=0$ and $A B C D \neq 0, E=0$ and $A B C D \neq 0, A=B=0$ and $C D E \neq 0, A=C=0$ and $B D E \neq 0, A=D=0$ and $B C E \neq 0, A=E=0$ and $B C D \neq 0, B=C=0$ and $A D E \neq 0, B=D=0$ and $A C E \neq 0, B=E=0$ and $A C D \neq 0, C=D=0$ and $A B E \neq 0, C=E=0$ and $A B C \neq 0, D=E=0$ and $A B D \neq 0, A=B=C=0$ and $D E \neq 0$, $A=B=D=0$ and $C E \neq 0, A=B=E=0$ and $C D \neq 0, A=C=D=0$ and $B E \neq 0, A=C=E=0$ and $B D \neq 0, A=D=E=0$ and $B C \neq 0$, $B=C=D=0$ and $A E \neq 0, B=C=E=0$ and $A D \neq 0, B=D=E=0$ and $A C \neq 0, C=D=E=0$ and $A B \neq 0, A=B=C=D=0$ and 
$E \neq 0, A=B=C=E=0$ and $D \neq 0, A=B=D=E=0$ and $C \neq 0$, $A=C=D=E=0$ and $B \neq 0, B=C=D=E=0$ and $A \neq 0)$. In this way we obtain 93 combinations. If we eliminate the cases where the singular points are not isolated, we obtain a total of 67 normal forms, taking into account the duplication due to the double sign.

If $a b A \neq 0$ and $b A>0$, then one can define $\alpha, \beta, \gamma$ as $\alpha=a \beta / b, \beta^{3}=b^{4} A / a^{5}, \gamma=$ $a$, in order to reduce the transformed coefficients as $a / \gamma=1, \alpha b /(\beta \gamma)=1$ and $\beta A /\left(\alpha^{4} \gamma\right)=1$. Therefore the resulting vector field writes $\left(\bar{x}+\bar{y}, \bar{x}^{3}+\bar{B} \bar{x}^{2} \bar{y}+\right.$ $\left.\bar{C} \bar{x} y^{2}+\bar{D} \bar{y}^{3}\right)$ with $\bar{B}=a B /(b A), \bar{C}=a^{2} C /\left(b^{2} A\right), \bar{D}=a^{3} D /\left(b^{3} A\right), \bar{E}=a^{4} D /\left(b^{4} A\right)$. Dropping the bars, we obtain the vector field $X_{1}$. In a similar way one can prove the 67 other cases.

In order to understand the minus sign in $X_{44}$ with respect to $X_{43}$, we remark that $\alpha=\beta \gamma / b, \beta^{3}=b^{4} A / \gamma^{5}$ and $\gamma^{4}=b^{4} A / E$ is a consequence of having taken $\bar{E}=1$, which happens in $X_{44}$, meaning that $A E>0$. If $A E<0$, then $\gamma^{4}=-b^{4} A / E$, leading to $X_{45}$. A similar situation happens to the forms $X_{46}$ and $X_{47}$ corresponding respectively to $A C>0$ and $A C<0$; to the forms $X_{62}$ and $X_{63}$, corresponding respectively to $C E>0$ and $C E<0$; and to the forms $X_{65}$ and $X_{66}$, corresponding respectively to $A E>0$ and $A E<0$.

From Proposition 2 we see that the study of the semi-homogeneous vector fields $\left(a x+b y, A x^{4}+B x^{3} y+C x^{2} y^{2}+D x y^{3}+E y^{4}\right)$ has been reduced to the analysis of semi-homogeneous vector fields depending at most of four parameters.

\section{Preliminary Definitions}

In this section we introduce the basic definitions and notations that we will need for the analysis of the local phase portraits of the finite and infinite singular points of the semi-homogeneous vector fields given in Proposition 2.

We denote by $\mathcal{P}_{n}\left(\mathbb{R}^{2}\right)$ the set of polynomial vector fields on $\mathbb{R}^{2}$ of the form $X(x, y)=(P(x, y), Q(x, y))$ where $P$ and $Q$ are real polynomials in the variables $x$ and $y$ of degree at most $n$ (with $n \in \mathbb{N}$ ). As usual $\mathbb{N}$ denotes the set of positive integers.

3.1. Singular points. A point $q \in \mathbb{R}^{2}$ is said to be a singular point of the vector field $X$ if $P(q)=Q(q)=0$. We recall first some results which hold when $P$ and $Q$ are analytic functions in a neighborhood of $q$.

If $\Delta=P_{x}(q) Q_{y}(q)-P_{y}(q) Q_{x}(q)$ and $T=P_{x}(q)+Q_{y}(q)$, then the singular point $q$ is said to be non-degenerate if $\Delta \neq 0$. Then $q$ is an isolated singular point. Moreover, $q$ is a saddle if $\Delta<0$, a node if $T^{2} \geq 4 \Delta>0$ (stable if $T<0$, unstable if $T>0$ ), a focus if $4 \Delta>T^{2}>0$ (stable if $T<0$, unstable if $T>0$ ), and either a weak focus or a center if $T=0<\Delta$; for more details see [3] p. 183.

A singular point $q$ is elementary if $\Delta=0$ and $T \neq 0$, and $q$ is isolated in the set of all singular points. In the next proposition we summarize the results on elementary singular points that we shall need in this paper. For a proof see Theorem 65 of [3].

Proposition 3. Let $(0,0)$ be an isolated point of the vector field $(F(x, y), y+$ $G(x, y))$, where $F$ and $G$ are analytic functions in a neighborhood of the origin starting in quadratic terms in the variables $x$ and $y$. Let $y=g(x)$ be the solution of the equation $y+G(x, y)=0$ in a neighborhood of $(0,0)$. Assume that the development of the function $f(x)=F(x, g(x))$ is of the form $f(x)=\mu x^{m}+H O T$ (Higher Order Terms), where $m \geq 2$ and $\alpha \neq 0$. When $m$ is odd then $(0,0)$ is either an 
unstable node or a saddle depending if $\mu>0$ or $\mu<0$ respectively. In the case of the saddle the stable separatrices are tangent to the $x$-axis. If $m$ is even, then $(0,0)$ is a saddle-node, i.e. the singular point is formed by the union of two hyperbolic sectors with one parabolic sector. The stable separatrix is tangent to the positive (repectively negative) $x$-axis at $(0,0)$ according to $\mu<0$ (respectively $\mu>0$ ). The two unstable separatrices are tangent to the $y$-axis at $(0,0)$.

When $\Delta=T=0$ but the Jacobian matrix at $q$ is not the zero matrix and $q$ is isolated in the set of all singular points, we say that $q$ is nilpotent. Now we summarize the results on nilpotent singular points that we shall need. For details see $[2]$.

Proposition 4. Let $(0,0)$ be an isolated singular point of the vector field $(y+$ $F(x, y), G(x, y))$, where $F$ and $G$ are analytic functions in a neighborhood of the origin starting in quadratic terms in the variables $x$ and $y$. Let $y=f(x)$ be the solution of the equation $y+F(x, y)=0$ in a neighborhood of $(0,0)$. Assume that the development of the function $G(x, f(x))$ is of the form $G(x, f(x))=K x^{\kappa}+H O T$ and $\Phi(x) \equiv(\partial F / \partial x+\partial G / \partial y)(x, f(x))=L x^{\lambda}+H O T$ with $K \neq 0, \kappa \geq 2$ and $\lambda \geq 1$. Then the following statements hold.

(1) If $\kappa$ is even and

(1.a) $\kappa>2 \lambda+1$, then the origin is a saddle-node. Moreover the saddlenode has one separatrix tangent to the semi-axis $x<0$, and the other two separatrices tangent to the semi-axis $x>0$.

(1.b) $\kappa<2 \lambda+1$ or $\Phi \equiv 0$, then the origin is a cusp, i.e. a singular point formed by the union of two hyperbolic sectors. Moreover, the cusp has two separatrices tangent to the positive $x$-axis.

(2) If $\kappa$ is odd and $K>0$, then the origin is a saddle. Moreover, the saddle has two separatrices tangent to the semi-axis $x<0$, and the other two tangent to the semi-axis $x>0$.

(3) If $\kappa$ odd, $K<0$ and

(3.a) $\lambda$ even, $\kappa=2 \lambda+1$ and $L^{2}+4 K(\lambda+1) \geq 0$, or $\lambda$ even and $\kappa>2 \lambda+1$, then the origin is a stable (unstable) node if $L<0(L>0)$, having all the orbits tangent to the $x$-axis at $(0,0)$.

(3.b) $\lambda$ odd, $\kappa=2 \lambda+1$ and $L^{2}+4 K(\lambda+1) \geq 0$, or $\lambda$ odd and $\kappa>2 \lambda+1$ then the origin is an elliptic-saddle, i.e. a singular point formed by the union of one hyperbolic sector and one elliptic sector. Moreover, one separatrix of the elliptic-saddle is tangent to the semi-axis $x<0$, and the other to the semi-axis $x>0$.

(3.c) $\kappa=2 \lambda+1$ and $L^{2}+4 K(\lambda+1)<0$, or $\kappa<2 \lambda+1$, then the origin is a focus and if $\Phi(x) \equiv 0$, then the origin is a center.

Finally if the Jacobian matrix at the singular point $q$ is identically zero, and $q$ is isolated inside the set of all singular points, then we say that $q$ is linearly zero. The study of its local phase portrait needs a special treatment (directional blow-ups), see for more details [5].

\section{Poincaré Compactification}

Let $X \in \mathcal{P}_{n}\left(\mathbb{R}^{2}\right)$ be any planar vector field of degree $n$. The Poincaré compactified vector field $p(X)$ corresponding to $X$ is an analytic vector field induced on $\mathbb{S}^{2}$ as follows (see, for instance [12]). Let $\mathbb{S}^{2}=\left\{y=\left(y_{1}, y_{2}, y_{3}\right) \in \mathbb{R}^{3}: y_{1}^{2}+y_{2}^{2}+y_{3}^{2}=1\right\}$ 
(the Poincaré sphere) and $T_{y} \mathbb{S}^{2}$ be the tangent space to $\mathbb{S}^{2}$ at point $y$. Consider the central projection $f: T_{(0,0,1)} \mathbb{S}^{2} \rightarrow \mathbb{S}^{2}$. This map defines two copies of $X$, one in the northern hemisphere and the other in the southern hemisphere. Denote by $X^{\prime}$ the vector field $D f \circ X$ defined on $\mathbb{S}^{2}$ except on its equator $\mathbb{S}^{1}=\left\{y \in \mathbb{S}^{2}: y_{3}=0\right\}$. Clearly $\mathbb{S}^{1}$ is identified to the infinity of $\mathbb{R}^{2}$. In order to extend $X^{\prime}$ to a vector field on $\mathbb{S}^{2}$ (including $\mathbb{S}^{1}$ ) it is necessary that $X$ satisfies suitable conditions. In the case that $X \in \mathcal{P}_{n}\left(\mathbb{R}^{2}\right), p(X)$ is the only analytic extension of $y_{3}^{n-1} X^{\prime}$ to $\mathbb{S}^{2}$. On $\mathbb{S}^{2} \backslash \mathbb{S}^{1}$ there are two symmetric copies of $X$, and knowing the behaviour of $p(X)$ around $\mathbb{S}^{1}$, we know the behaviour of $X$ at infinity. The projection of the closed northern hemisphere of $\mathbb{S}^{2}$ on $y_{3}=0$ under $\left(y_{1}, y_{2}, y_{3}\right) \longmapsto\left(y_{1}, y_{2}\right)$ is called the Poincaré disc, and it is denoted by $\mathbb{D}^{2}$. The Poincaré compactification has the property that $\mathbb{S}^{1}$ is invariant under the flow of $p(X)$.

In the rest of this paper we say that two polynomial vector fields $X$ and $Y$ on $\mathbb{R}^{2}$ are topologically equivalent if there exists a homeomorphism on $\mathbb{S}^{2}$ preserving the infinity $\mathbb{S}^{1}$ carrying orbits of the flow induced by $p(X)$ into orbits of the flow induced by $p(Y)$, preserving or reversing simultaneously the sense of all orbits.

As $\mathbb{S}^{2}$ is a differentiable manifold, for computing the expression for $p(X)$, we can consider the six local charts $U_{i}=\left\{y \in \mathbb{S}^{2}: y_{i}>0\right\}$, and $V_{i}=\left\{y \in \mathbb{S}^{2}: y_{i}<0\right\}$ where $i=1,2,3$; and the diffeomorphisms $F_{i}: U_{i} \rightarrow \mathbb{R}^{2}$ and $G_{i}: V_{i} \rightarrow \mathbb{R}^{2}$ for $i=1,2,3$ are the inverses of the central projections from the planes tangent at the points $(1,0,0),(-1,0,0),(0,1,0),(0,-1,0),(0,0,1)$ and $(0,0,-1)$ respectively. If we denote by $z=\left(z_{1}, z_{2}\right)$ the value of $F_{i}(y)$ or $G_{i}(y)$ for any $i=1,2,3$ (so $z$ represents different things according to the local charts under consideration), then some easy computations give for $p(X)$ the following expressions:

$$
\begin{aligned}
& z_{2}^{n} \Delta(z)\left(Q\left(\frac{1}{z_{2}}, \frac{z_{1}}{z_{2}}\right)-z_{1} P\left(\frac{1}{z_{2}}, \frac{z_{1}}{z_{2}}\right),-z_{2} P\left(\frac{1}{z_{2}}, \frac{z_{1}}{z_{2}}\right)\right) \quad \text { in } \quad U_{1}, \\
& \text { (2) } z_{2}^{n} \Delta(z)\left(P\left(\frac{z_{1}}{z_{2}}, \frac{1}{z_{2}}\right)-z_{1} Q\left(\frac{z_{1}}{z_{2}}, \frac{1}{z_{2}}\right),-z_{2} Q\left(\frac{z_{1}}{z_{2}}, \frac{1}{z_{2}}\right)\right) \quad \text { in } \quad U_{2} \text {, } \\
& \Delta(z)\left(P\left(z_{1}, z_{2}\right), Q\left(z_{1}, z_{2}\right)\right) \quad \text { in } \quad U_{3},
\end{aligned}
$$

where $\Delta(z)=\left(z_{1}^{2}+z_{2}^{2}+1\right)^{-\frac{1}{2}(n-1)}$. The expression for $V_{i}$ is the same as that for $U_{i}$ except for a multiplicative factor $(-1)^{n-1}$. In these coordinates for $i=1,2$, $z_{2}=0$ always denotes the points of $\mathbb{S}^{1}$. In what follows we omit the factor $\Delta(z)$ by rescaling the vector field $p(X)$. Thus we obtain a polynomial vector field in each local chart.

\section{Finite SINGUlar POINTS}

As $P$ and $Q$ are homogeneous polynomials, they only can intersect at the origin or at a straight line through the origin. So the finite singular points of $X=(P, Q)$ are either the origin or a straight line through the origin. We do not consider in this paper the second possibility. The next Proposition 5 will characterize the local phase portrait at the origin of $\mathrm{X}$.

Proposition 5. We denote by $X_{i}$, for $i=1,2, \ldots, 67$, the vector fields of Proposition 2.

(a) The origin is an elementary saddle-node for $X_{i}$ with $i \in\{1-31,50-67\}$

(b) The origin is a cusp for $X_{i}$ with $i \in\{32-49\}$

Before proving Proposition 5 we need the following auxiliary lemmas. 
Lemma 6. Let $X$ be any planar quartic vector field of the form $\left(a x+b y, A x^{4}+\right.$ $\left.B x^{3} y+C x^{2} y^{2}+D x y^{3}+E y^{4}\right)$, then the origin is elementary if $a \neq 0$, and is linearly zero if $a=0$.

Proof. At the origin the Jacobian matrix writes

$$
\left(\begin{array}{ll}
a & b \\
0 & 0
\end{array}\right)
$$

By inspection of (3) the lemma follows immediately. Note that since $P$ must be linear, the case $a=b=0$ is not possible.

Lemma 7. Consider the vector field $\left(x+b y, A x^{4}+B x^{3} y+C x^{2} y^{2}+D x y^{3}+E y^{4}\right)$, then the local phase portrait around the origin is an elementary saddle-node with two hyperbolic sectors having the two unstable separatrices tangent to the $x$-axis and the stable one tangent to the half-straight line $x+b y=0$, with $y<0$ (respectively $y>0$ ) when $A b^{4}-B b^{3}+C b^{2}-D b+E>0$ (respectively $\left.<0\right)$.

Proof. In order to apply Proposition 3 to our vector field at the origin, we need to do the change of variables $x_{1}=y, x_{2}=x+b y$. Then the vector field becomes into the form $\left.F\left(x_{1}, x_{2}\right), x_{2}+G\left(x_{1}, x_{2}\right)\right)$ where

$$
\begin{aligned}
F\left(x_{1}, x_{2}\right)= & \left.\left(A b^{4}-B b^{3}+C b^{2}-D b+E\right) x_{1}^{4}-\left(4 A b^{3}-3 B b^{2}+2 C b-D\right) x_{1}^{3} x_{2}\right)+ \\
& \left(6 A b^{2}-3 B b+C\right) x_{1}^{2} x_{2}^{2}-(4 A b-B) x_{1} x_{2}^{3}+A x_{2}^{4}
\end{aligned}
$$

and

$$
\begin{aligned}
G\left(x_{1}, x_{2}\right)= & \left.b\left(A b^{4}-B b^{3}+C b^{2}-D b+E\right) x_{1}^{4}-b\left(4 A b^{3}-3 B b^{2}+2 C b-D\right) x_{1}^{3} x_{2}\right)+ \\
& b\left(6 A b^{2}-3 B b+C\right) x_{1}^{2} x_{2}^{2}-b(4 A b-B) x_{1} x_{2}^{3}+A b x_{2}^{4} .
\end{aligned}
$$

Let $x_{2}=g\left(x_{1}\right)=b\left(A b^{4}-B b^{3}+C b^{2}-D b+E\right) x_{1}^{4}+H O T$ the solution of $x_{2}+$ $G\left(x_{1}, x_{2}\right)=0$ in a neighborhood of $(0,0)$. Then $f\left(x_{1}\right)=F\left(x_{1}, g\left(x_{1}\right)\right)=\left(A b^{4}-\right.$ $\left.B b^{3}+C b^{2}-D b+E\right) x_{1}^{2}+H O T$. Hence, by Proposition 3, the lemma follows.

Lemma 8. Consider the vector field $\left(y, A x^{4}+B x^{3} y+C x^{2} y^{2}+D x y^{3}+E y^{4}\right)$, then the straight line $x+$ by is formed by singular points when $A b^{4}-B b^{3}+C b^{2}-D b+E=0$.

Proof. The vector field can easily be written as $(x+b y),(x+b y)\left(A x^{3}+(B-A b) x^{2} y+\right.$ $\left.\left(A b^{2}+C-B b\right) x y^{2}+\left(B b^{2}-A b^{3}+D-C b\right) y^{3}\right)$ proving the lemma.

Lemma 9. Consider the vector field $\left(y, A x^{4}+B x^{3} y+C x^{2} y^{2}+D x y^{3}+E y^{4}\right)$, then the origin is a cusp having its two separatrices tangent to the positive $x$-axis.

Proof. We can apply Proposition 4 to the origin of our vector field $(y+F(x, y), G(x, y))=$ $\left(y, x^{4}+B x^{3} y+C x^{2} y^{2}+D x y^{3}+E y^{4}\right)$. Then $y=f(x)=0, G(x, 0)=x^{4}=K x^{\kappa}$, and $(\partial F / \partial x+\partial G / \partial y)(x, 0)=B x^{3}=L x^{\lambda}$. Since $\kappa=4$ is even and $\kappa<2 \lambda+1=7$, from Proposition 4(1.b) it follows the lemma.

The proof of Proposition 5 follows immediately of Lemmas 7 and 8 


\section{INFINITE SINGULAR POINTS}

The expressions (1) and (2) for the Poincaré compactification $p(X)$ of $X=$ $\left(a x+b y, A x^{4}+B x^{3} y+C x^{2} y^{2}+D x y^{3}+E y^{4}\right)$ write as follows

(4) $\quad\left(A+B z_{1}+C z_{1}^{2}+D z_{1}^{3}+E z_{1}^{4}-z_{1} z_{2}^{3}\left(a+b z_{1}\right),-z_{2}^{4}\left(a+b z_{1}\right)\right)$ in $U_{1}$, $\left(-E z_{1}-D z_{1}^{2}-C z_{1}^{3}+b z_{2}^{3}-B z_{1}^{4}+a z_{1} z_{2}^{3}-A z_{1}^{5},-E z_{2}-D z_{1} z_{2}-C z_{1}^{2} z_{2}\right.$

$$
\left.\left.-B z_{1}^{3} z_{2}-A z_{1}^{4} z_{2}\right)\right) \text { in } U_{2}
$$

We remark that the singular points of $p(X)$ are symmetric with respect to the origin of the Poncaré sphere. Therefore in order to study the infinite singular points, we can restrict our analysis to the infinite singular points on the local charts $U_{1}$ and $U_{2}$. However, from the definitions of the local charts the unique infinite critical point which may belong to $\left(U_{2} \bigcup V_{2}\right) \backslash\left(U_{1} \bigcup V_{1}\right)$ are the origins of $U_{2}$ and $V_{2}$. So the infinite singular points of $X$ are the points $\left(z_{1}, 0\right)$ of $U_{1}$ such that $A+B z_{1}+C z_{1}^{2}+D z_{1}^{3}+E z_{1}^{4}=0$, and the $(0,0)$ of $U_{2}$.

In order to study the local behavior at infinite singular points, we shall need their linear parts given by the Jacobian matrices which are of the form

$$
\left(\begin{array}{cc}
B+2 C z_{1}+3 D z_{1}^{2}+4 E z_{1}^{3} & 0 \\
0 & 0
\end{array}\right) \text { and }\left(\begin{array}{cc}
-E & 0 \\
0 & -E
\end{array}\right)
$$

at the points $\left(z_{1}, 0\right) \in U_{1}$ and $(0,0) \in U_{2}$ respectively.

6.1. The origin of the local chart $U_{2}$ as infinite singular point. From (6) the origin of the local chart $U_{2}$ is non-degenerate if $E \neq 0$ and linearly zero if $E=0$.

Proposition 10. We denote by $X_{i}$, for $i=1,2, \ldots, 67$, the vector fields of Proposition 2. The origin of the local chart $U_{2}$ is

(a) a stable node if $E>0$,

(b) an unstable node if $E<0$,

(c) an elliptic-saddle if $E=0$ and $D \neq 0$. If $D>0$ (respectively $D<0$ ) the elliptic sector is on $z_{2}>0$ (respectively $z_{2}<0$ ), and the stable separatrix coincides locally with the positive (respectively negative) $z_{1}$-axis,

(d) a stable node if $D=E=0$ and $C>0$,

(e) an unstable node if $D=E=0$ and $C<0$,

(f) an elliptic-saddle if $C=D=E=0$ and $B \neq 0$. If $B>0$ (respectively $B<0$ ) the elliptic sector is on $z_{2}>0$ (respectively $\left.z_{2}<0\right)$, and the stable separatrix coincides locally with the positive(respectively negative) $z_{1}$-axis,

(g) a stable node if $B=C=D=E=0$ and $A>0$,

(h) an unstable node if $B=C=D=E=0$ and $A<0$.

Proof. If $E \neq 0$ from (6) the origin of $U_{2}$ is a non-degenerated singular point. Therefore from Section 3.1 statements (a) and (b) follow.

If we assume that $E=0$ and $A B C D \neq 0$ we note that $b=1$, otherwise $b=0$ and the vector field $X$ would have the straight line $x=0$ formed by singular points. Now the vector field (5) has a linearly zero singular point. Doing the directional blow-up $\left(z_{1}, z_{2}\right) \rightarrow\left(z_{1}, z_{3}\right)$ with $z_{3}=z_{2} / z_{1}$, the vector field (5) becomes

$$
\left(-D z_{1}^{2}-C z_{1}^{3}-B z_{1}^{4}+z_{1}^{3} z_{3}^{3}+a z_{1}^{4} z_{3}^{3}-A z_{1}^{5},-z_{3}^{4} z_{1}^{2}-a z_{3}^{4} z_{1}^{3}\right)
$$

and rescaling by the factor $z_{1}$

$$
\left(-D-C z_{1}-B z_{1}^{2}+z_{1} z_{3}^{3}+a z_{1}^{2} z_{3}^{3}-A z_{1}^{3},-z_{3}^{4}-a z_{3}^{4} z_{1}\right) .
$$


Going back through the changes of variables we obtain statement (c).

If we assume that $D=E=0$ and $A B C \neq 0$ then the directional blow-up $\left(z_{1}, z_{2}\right) \rightarrow\left(z_{1}, z_{3}\right)$ on the vector field (5) becomes

$$
\left(-C z_{1}^{3}-B z_{1}^{4}+z_{1}^{3} z_{3}^{3}+a z_{1}^{4} z_{3}^{3}-A z_{1}^{5},-z_{3}^{4} z_{1}^{2}-a z_{3}^{4} z_{1}^{3}\right),
$$

and rescaling by the factor $-C z_{1}^{2}$

$$
\left(z_{1}+\frac{B}{C} z_{1}^{2}-\frac{1}{C} z_{1} z_{3}^{3}-\frac{a}{C} z_{1}^{2} z_{3}^{3}+\frac{A}{C} z_{1}^{3}, \frac{1}{C} z_{3}^{4}+\frac{a}{C} z_{3}^{4} z_{1}\right) .
$$

This vector field has only one singular point in $z_{1}=0$, the origin. This point is a saddle-node in according to Proposition 3. Going back through the changes of variables we obtain statements (d) and (e).

If we assume that $C=D=E=0$ and $A B \neq 0$ then the directional blow-up $\left(z_{1}, z_{2}\right) \rightarrow\left(z_{1}, z_{3}\right)$ on the vector field (5) becomes

$$
\left(-B z_{1}^{4}-A z_{1}^{5}+z_{1}^{3} z_{3}^{3}+a z_{1}^{4} z_{3}^{3},-z_{3}^{4} z_{1}^{2}-a z_{3}^{4} z_{1}^{3}\right),
$$

and rescaling by the factor $z_{1}^{2}$

$$
\left(-B z_{1}^{2}-A z_{1}^{3}+z_{1} z_{3}^{3}+a z_{1}^{2} z_{3}^{3},-z_{3}^{4}-a z_{3}^{4} z_{1}\right) .
$$

Now the vector field (7) has at the origin a linearly zero singular point. Doing the directional blow-up $\left(z_{1}, z_{3}\right) \rightarrow\left(z_{1}, z_{4}\right)$ with $z_{4}=z_{3} / z_{1}$, the vector field (7) becomes

$$
\left(-B z_{1}^{2}-A z_{1}^{3}+z_{1}^{4} z_{4}^{3}+a z_{1}^{5} z_{4}^{3}, B z_{1} z_{4}+A z_{1}^{2} z_{4}-2 z_{1}^{3} z_{4}^{4}-2 a z_{1}^{4} z_{4}^{4}\right)
$$

and rescaling by the factor $z_{1}$

$$
\left(-B z_{1}-A z_{1}^{2}+z_{1}^{3} z_{4}^{3}+a z_{1}^{4} z_{4}^{3}, B z_{4}+A z_{1} z_{4}-2 z_{1}^{2} z_{4}^{4}-2 a z_{1}^{3} z_{4}^{4}\right),
$$

which is a saddle. Going back through the changes of variables we obtain statement (f).

Finally if we assume that $B=C=D=E=0$ then the directional blow-up $\left(z_{1}, z_{2}\right) \rightarrow\left(z_{1}, z_{3}\right)$ on the vector field (5) becomes

$$
\left(-A z_{1}^{5}+z_{1}^{3} z_{3}^{3}+a z_{1}^{4} z_{3}^{3},-z_{3}^{4} z_{1}^{2}-a z_{3}^{4} z_{1}^{3}\right)
$$

and rescaling by the factor $z_{1}^{2}$

$$
\left(-A z_{1}^{3}+z_{1} z_{3}^{3}+a z_{1}^{2} z_{3}^{3},-z_{3}^{4}-a z_{3}^{4} z_{1}\right) .
$$

Now the vector field (8) has at the origin a linearly zero singular point. Doing the directional blow-up $\left(z_{1}, z_{3}\right) \rightarrow\left(z_{1}, z_{4}\right)$ with $z_{4}=z_{3} / z_{1}$, the vector field (8) becomes

$$
\left(-A z_{1}^{3}+z_{1}^{4} z_{4}^{3}+a z_{1}^{5} z_{4}^{3}, A z_{1}^{2} z_{4}-2 z_{1}^{3} z_{4}^{4}-2 a z_{1}^{4} z_{4}^{4}\right)
$$

and rescaling by the factor $z_{1}^{2}$

$$
\left(-A z_{1}+z_{1}^{2} z_{4}^{3}+a z_{1}^{3} z_{4}^{3}, A z_{4}-2 z_{1} z_{4}^{4}-2 a z_{1}^{2} z_{4}^{4}\right),
$$

which is a saddle. Going back through the changes of variables we obtain statements (g) and (h). 
6.2. The infinite singular points of the local chart $U_{1}$. From (4) the infinite singular points of the local chart $U_{1}$ of $X=\left(a x+b y, A x^{4}+B x^{3} y+C x^{2} y^{2}+D x y^{3}+\right.$ $\left.E y^{4}\right)$ satisfy $F\left(z_{1}\right)=A+B z_{1}+C z_{1}^{2}+D z_{1}^{3}+E z_{1}^{4}=0$. We define

$$
\omega=-3 k^{4} E^{2}\left(C^{2}\left(4 C E-D^{2}\right)\left(4 A C-B^{2}\right)-9 A^{2} D^{2}\left(3 D^{2}-16 C E\right)-\right.
$$

$128 A^{2} E^{2}\left(C^{2}-2 A E\right)-2 A B C D\left(40 C E-9 D^{2}\right)+2 D B^{3}\left(9 C E-2 D^{2}\right)+$ $\left.6 A B^{2} E\left(24 C E-D^{2}\right)-3 B E^{2}\left(9 B^{3}+64 D A^{2}\right)\right)$ and

$$
\Delta_{ \pm}=\left(D+E r_{ \pm}\right)\left(\left(D+E r_{ \pm}\right) r_{ \pm}-16 E s_{l}\right)+16 E B, \quad(l=1,2,3)
$$

where

$$
\begin{aligned}
& k=2 / 3 E /\left(4 A C E-A D^{2}-B^{2} E\right), \\
& r_{ \pm}= \pm\left(8 E^{2} s_{l}+D^{2}-4 C E\right)^{1 / 2} / E \\
& s_{1}=u+v \\
& s_{2}=\left(-u-v+3^{1 / 2}(u-v) i\right) / 2, \\
& s_{3}=\left(-u-v-3^{1 / 2}(u-v) i\right) / 2, \\
& u=\left(-q+\omega^{1 / 2}\right)^{1 / 3} \\
& v=\left(-q-\omega^{1 / 2}\right)^{1 / 3}, \\
& q=k^{3}\left(9 A B C D\left(4 E C-D^{2}\right)-30 A B^{2} E\left(6 E C-D^{2}\right)+\right. \\
& 9 A^{2}\left(-4 E C+D^{2}\right)\left(-8 E C+3 D^{2}\right)+B^{3} D\left(2 D^{2}-9 E C\right)+ \\
& \left.3 B E^{2}\left(9 B^{3}+32 A^{2} D\right)-128 A^{3} E^{3}\right) / 2 .
\end{aligned}
$$

Proposition 11. If $E \neq 0$ then $F$ has four simple real roots if $\Delta_{ \pm}>0$; two simple real roots and one double real roots if $\Delta_{+}>0$ and $\Delta_{-}=0$, (or alternatively $\Delta_{+}=0$ and $\Delta_{-}>0$ ); one double real root if $\Delta_{+}<0$ and $\Delta_{-}=0$, (or alternatively $\Delta_{+}=0$ and $\Delta_{-}<0$ ); two simple real roots if $\Delta_{+}>0$ and $\Delta_{-}<0$, (or alternatively $\Delta_{+}<0$ and $\left.\Delta_{-}>0\right)$ and zero real roots if $\Delta_{ \pm}<0$,

Proof. $F\left(z_{1}\right)$ has the same roots as the two second order equations $-2 B+(D+$ $\left.E r_{ \pm}\right)\left(r_{ \pm} z_{1}+2 s_{l}\right)+2 E r_{ \pm} z_{1}^{2}=0$, where $s_{l}$ is a real root of the third order equation $A\left(4 C E-D^{2}\right)-B^{2} E+2 E(B D-4 A E) s_{l}-4 C E^{2} s_{l}^{2}+8 E^{3} s_{l}^{3}=0$ (see for more details [1]). Using this result the Proposition follows.

\section{REFERENCES}

[1] M. Abramowitz, I. A. Stegun "Handbook of Mathematical Functions", Dover, 1965.

[2] A. F. AndreEv, "Investigation of the behaviour of the integral curves of a system of two differential equations in the neighborhood of a singular point", Translation of Amer. Math. Soc. 8 (1958), 183-207.

[3] A. A. Andronov, E. A. Leontovich, I. I. Gordon, And A. L. Maier, "Qualitative Theory of Second-Order Dynamical Systems", Wiley, New York, 1973.

[4] J. Argemí, "Sur les points singuliers multiples de systmes dynamiques dans $R^{2}$ ", Annali di Matematica Pura ed Applicata, Serie IV 79 (1968), 35-70.

[5] V. I. Arnold and Y. S. Ilyashenko, "Dynamical Systems I, Ordinary Differential Equations". Encyclopaedia of Mathematical Sciences, Vols 1-2, Springer-Verlag, Heidelberg, 1988.

[6] L. CAIRó AND J. Llibre, "Phase portraits of planar semi-homogeneous vector fields-I", Nonlinear Analysis, Theory, Methods and Applications, 29 (1997), 783-811.

[7] L. Cairó And J. Llibre, "Phase portraits of planar semi-homogeneous vector fields-II", Nonlinear Analysis, Theory, Methods and Applications, 39 (2000), 351-363. 
[8] A. Cima And J. Llibre, "Algebraic and topological classification of the homogeneous cubic vector fields in the plane", J. of Math. Anal. and Appl. 147 (1990), 420-448.

[9] A. Cima, A. Gasull and F. Mañosas, "Cyclicity of a family of vector fields", J. of Math. Anal. and Appl. 196 (1995), 921-937.

[10] C. B. Collins, "Algebraic classification of homogeneous polynomial vector fields in the plane", preprint, University of Waterloo, 1993.

[11] T. DATE, "Classification and analysis of two-dimensional homogeneous quadratic differential equations systems", J. of Diff. Equations 32 (1979), 311-334.

[12] E. A. GonzÁLez, "Generic properties of polynomial vector fields at infinity", Trans. Amer. Math. Soc. 143 (1969), 201-222

[13] N. A. Korol, "The integral curves of a certain differential equation", (in Russian), Minsk. Gos. Ped. Inst. Minsk (1973), 47-51.

[14] J. Llibre, J. S. PÉrez del Río And J. A. Rodríguez, "Structural stability of planar homogeneous polynomial vector fields. Applications to critical points and to infinity", J. Differential Equations 125 (1996), 490-520.

[15] J. Llibre, J. S. PÉrez del Río And J. A. Rodríguez, "Structural stability of planar semi-homogeneous polynomial vector fields. Applications to critical points and to infinity", preprint, 1996.

[16] L. S. Lyagina, "The integral curves of the equation $y^{\prime}=\left(a x^{2}+b x y+c y^{2}\right) /\left(d x^{2}+e x y+f y^{2}\right)$ " (in Russian), Usp. Mat. Nauk, 6-2(42) (1951), 171-183.

[17] L. MARKus, "Quadratic differential equations and non-associative algebras", Annals of Mathematics Studies, Vol 45, Princeton University Press, 1960, pp 185-213.

[18] T. A. Newton " Two dimensional homogeneous quadratic differential systems", SIAM Review 20 (1978), 120-138.

[19] K. S. Sibirskit And N. I. VulPe, "Geometric classification of quadratic differential systems", Differential Equations 13 (1977), 548-556.

[20] E. V. Vdovina, "Classification of singular points of the equation $y^{\prime}=\left(a_{0} x^{2}+a_{1} x y+\right.$ $\left.a_{2} y^{2}\right) /\left(b_{0} x^{2}+b_{1} x y+b_{2} y^{2}\right)$ by Forster's method" (in Russian), Diff. Uravn. 20 (1984), 18091813.

MAPMO/CNRS-Departement de Mathématiques. Université d'Orleans, 45067 Orléans, Cedex 2, France

E-mail address: lcairo@wanadoo.fr

Departament de Matemàtiques, Universitat Autònoma de Barcelona, 08193 Bellaterra, BARCELONA, SpAiN

E-mail address: jllibre@mat.uab.cat 